\title{
Aligning Patient Preferences and Patient Care at the End of Life
}

\author{
Andrew Dunn, MD, FACP, FHM' and Evgenia Litrivis, $M D^{2}$ \\ 'Department of Medicine, Mount Sinai Medical Center, New York, NY, USA; ${ }^{2}$ Department of Geriatrics, Mount Sinai Medical Center, New York, \\ NY, USA.
}

J Gen Intern Med 26(7):681-2

DOI: $10.1007 / \mathrm{s} 11606-011-1738-1$

(C) Society of General Internal Medicine 2011

$\mathrm{S}$ tudies have shown that patients' priorities for quality care during advanced illness and at the end of life include consistent and reliable medical information, expert pain and symptom management, avoiding inappropriate prolongation of the dying process, relieving burdens on loved ones, and being prepared for death. ${ }^{1-4}$ Despite patients' focus on quality of life issues, the majority of current Medicare spending is allocated towards hospital care, ${ }^{1}$ and a significant proportion of this expenditure goes towards costly "cure-driven" interventions at the end of life. In an effort to deliver treatment plans that are concordant with patients' priorities, identification of patients' goals of care and alignment with medical delivery is critical.

Mounting evidence suggests that addressing goals of care is directly linked with higher quality. A recent study, for example, revealed that a lack of "end-of-life" discussions in the hospital setting was associated with escalations in care in the last weeks of life and worse overall rated quality of care ${ }^{4}$. In this issue of JGIM, Yuen and colleagues ${ }^{5}$ describe existing barriers and propose methods to facilitate earlier and more efficient discussions of cardiopulmonary resuscitation preferences in the inpatient setting.

To ensure high quality care during advanced illness and at the end of life, educational initiatives and assessment methods focused on improving communication skills coupled with system changes need to be implemented.

Guidelines for addressing cardiopulmonary resuscitation preferences with patients and surrogate decision makers have been formulated by experts in the field of palliative care. ${ }^{6-8}$ The recommended steps include discussing and clarifying any misconceptions about the current medical condition and prognosis, eliciting goals and values for care, and discussing code status in a manner that adheres to the criteria for informed consent and if necessary making a physician recommendation. Yet this strategy is rarely followed in practice ${ }^{9,10}$. While core measures for the management of common medical conditions, such as aspirin administration after a myocardial infarction, have improved standards of care, delineating the required key elements in this communication process would be an important step for accrediting bodies and hospitals.

Educating trainees will be essential in ensuring that these communication guidelines are utilized in patient encounters. Despite the fact that housestaff frequently address code status, these conversations are often brief, and do not elicit the patient's understanding of their prognosis or explore the

Published online June 3, 2011 patient's goals and values. Additionally, they rarely meet criteria for informed consent ${ }^{9}$. This deficiency is not limited to trainees. A study of attending hospitalists showed a similar poor performance $^{10}$. Data further reveal that inadequate training leads to discomfort on the part of interns and residents when discussing these very important issues ${ }^{11}$. Mandatory formal curricula addressing this important skill set would address the deficiencies of these conversations. An isolated noon conference didactic, however, will not be sufficient. Education training that employs evidence-based literature and active learning with opportunities for practice, and with timely and constructive feedback has been identified as a proven strategy for improving resident communication skills ${ }^{12}$. Just as housestaff must demonstrate proficiency in procedures such as central line placement, they too should demonstrate proficiency in key communication skills, including having an effective code status discussion. This can be achieved through direct observation of resident interactions with patients and family members at the bedside, in family meetings or through role play with simulated patients. Feedback on specific skill application would ideally be provided by faculty educators who have acquired and demonstrated skills in palliative care and physician-patient communication.

Communication initiatives should also target hospitalists, given their growing presence in the hospital. Hospitalists will need to arm themselves with the communication skill set necessary to negotiate goals of care and elicit code status preferences so that they can provide high quality care. Recognizing the specific knowledge base and skills necessary to care for hospitalized patients, the ABIM has created a focused practice in hospital medicine (FPHM) pathway for maintenance of certification for hospitalists. This pathway emphasizes key inpatient topics, including palliative care and communication. Encouraging hospitalists to complete the FPHM pathway would enhance knowledge and spur clinicians' to develop additional expertise in physician-patient communication.

Once code status is clarified, further efforts to improve its exportability across care sites and minimize transition errors will be critical. A key component will be the inclusion of code status and goals of care on discharge summaries. Clinicians should be prompted to have the necessary discussions, and if agreed upon by patients, to complete out of hospital DNRs or POLSTs (physician orders for life-sustaining treatments) so that patients' resuscitation preferences are adhered to out of the hospital setting as well.

Several national initiatives to enhance communication at the end of life have been hindered by a distorted emphasis on limiting health care rather than ensuring high quality patient centered care throughout life. Transforming the national debate on end-of-life care from a contentious discussion on health care rationing to one that focuses on ensuring that patients receive the care they want is necessary. Palliative care 
providers and clinicians who care for patients with serious and life-limiting illness will be critical in guiding these discussions. The appreciation that addressing resuscitation preferences and other treatment options will promote patient autonomy and high quality care will act as a great catalyst for change.

Communication with patients and families at the end of life remains poor and needs to improve for patients to receive the same high-quality patient-centered care they expect throughout other stages of life. For communication at the bedside to be enhanced in a systematic fashion, the national conversation surrounding end-of-life care must also improve.

Corresponding Author: Andrew Dunn, MD, FACP, FHM; Department of Medicine, Mount Sinai Medical Center, One Gustave L. Levy Place, Box 1087, New York, NY 10029, USA (e-mail: Andrew. dunn@mountsinai.org).

\section{REFERENCES}

1. Partnership for Solutions. Chronic Conditions: Making the Case for Ongoing Care. Baltimore: John Hopkins University, 2002
2. Frield M, Cassel C. Institute of Medicine (IOM Report). Approaching Death: Improving Care at the End of Life. Washington, DC: National Academy Press; 1997.

3. Steinhauser KE, Christakis NA, Clipp EC, et al. Factors considered important at the end of life by patients, family, physicians, and other care providers. JAMA. 2000;284:2476-82.

4. Wright A, Zhang MS, Ray A, et al. Associations between end-of-life discussions, patient mental health, medical care near death, and caregiver bereavement adjustment. JAMA. 2008;300:1665-73.4.

5. Yuen JK, Reid C, Fetters MD. Hospital do-not-resuscitate orders: why they have failed and how to fix them. J Gen Intern Med 2011; doi:101007/s11606-011-1632-x.

6. von Gunten CF. Discussing do-not-resuscitate status. J Clin Oncol. 2001;19:1576-81.

7. Back A, Arnold R, Tulsky J. Talking about dying: "Do not resuscitate" orders and goodbyes. New York: Cambridge University Press; 2009: 121-36.

8. von Gunten CF, Weissman DE. Discussing DNR Orders-Part 1, 2nd edn. Fast Facts and Concepts \#23. http://www.mcw.edu, End of Life/ Palliative Education Resource Center, Medical College of Wisconsin, 2005.

9. Tulsky JA, Chesnet MA, Lo B. How do medical residents discuss resuscitation with patients? J Gen Intern Med. 1995;10(8):436-442.

10. Anderson W, Chase R, et al. Code status discussions between attending hospitalist physicians and medical patients at hospital admission. J Gen Intern Med. 2010;26(4):359-66.

11. Ury, WA, Berkman, CS. Assessing medical students' training in end-oflife communication: A survey of interns at one urban teaching hospital. Acad Med. 2003;78(5).

12. Gordon, B. Assessment of Physician-Patient Communication. Presented at ACGME/ABMS Assessment of Physician Patient Communication Conference. 2002 as cited in Advancing Education in Interpersonal and Communication Skills, ACGME Outreach Project. 